\title{
Survey on Quality of Service Recommendation
}

\author{
Dinkar Jadhav ${ }^{1}$, Prof. B.S. Satpute ${ }^{2}$ \\ Research Scholar, Dept. of Computer Science, DYP College of Engineering, Pimpri, Pune, India ${ }^{1}$ \\ Professor, Dept. of Computer Science, DYP College of Engineering, Pimpri, Pune, India ${ }^{2}$
}

\begin{abstract}
Tremendous popularity of the ecommerce applications increased now days. Numerous amounts of reviews and ratings are available on the sites. Such as comments, reviews description of the local service. This type of information is valuable for the new user who judges the product online to make their decision. Sometimes this information may be having problem to recommend user about product recommendation because of the lack of reviews and rating of particular product. If any product has two rating one 5 and other is 2 they only have two ratings. So while recommending this product just finds average of ratings and show to user. This not provides quality of service of the recommendation. This is not enough to extract public opinions. To provide quality of service to the recommendation have to improve overall evaluation of rating. Find the trust of the user service rating and feature. Extract the overall rating and trust of service by combining the service rating to providing quality of service.
\end{abstract}

Keywords: Data Mining, Recommendation system, Quality of Service Evaluation, Social Network.

\section{INTRODUCTION}

Now day's user receiving digitalized information from the internet. The volume of information is large. Users share their experience about items on the sites. The recommendation system exploits the information and recommends user preferred item and products [3]. Some social media applications give ideas to recommend services to user. In many area such as music, Videos, product/item or the travel places recommendation work by extraction information available on the site [3][4][11][14][15]. Collaborative filtering model are presented to improve accuracy of the recommendation. In recommendation system user individual preference is the major influence on the recommendation. User personal interest, similarity and interpersonal influence are combined in the recommendation [4]. If we are taking example of ecommerce application there is tremendous amount of data present on the site in the digital format. This data is in reviews, comments and the rating given by the users. Opinion of any product present on the site. This information guides to the new users, who want to buy something from the site. Numbers of user are also relying on these feedbacks from other users given. They judge the product based on the review and rating available on the site. Sometimes this information may be not true about any particular product. The information given is misguides user about that product or accept it as a correct. Such problem state the if any item has given only two ratings by other user, 5 star rating and 2 star rating generally we average this and recommends but it is wrong approach over the other large number of ratings. In these recommendation system focus on the personalize recommendation or personalized rating prediction. One of the main techniques in the personalized [6][8][15] rating prediction is matrix factorization . Matrix factorization extracts the user item rating matrix and recommends based on the similarity of the user who given same rating to the single item [8]. This system simply calculates average between item ratings and predict as a recommendation. Another technique is the collaborative filtering algorithm this also predict rating by calculating average rating of items based on the similarity and correlation between user [1][2][9][14]. Aims to recommends based prior user interaction [9]. This system predicts item on the base of previously rated by other users [1]. There are two general types of collaborative filtering memory based filtering and model based filtering [1]. In the memory based collaborative filtering predicts item based on the overall average rating of the item previously rated by the user [1]. This approach have problem while providing quality of recommendation to the user.

\section{PROBLEM CONTEXT}

In second section we explain our work briefly to set of methodology and overview of Recommendation techniques and approaches.

\section{A. Background}

Bayesian latent variable models users' latent interest and items latent topic. For improve rating extension enhance user dependent and item dependent biases [10]. They are lots of research have been done on the collaborative recommendation system to deal with the personalized recommendation [1]. Collaborative filtering based on graph uses similarity of graph vertices to make recommendation. In this method user item relationship finds by exploiting graph theory and correlated graph [2]. Based on the oblique projection, multi-domain collaborative filtering utilized for spatial domain and polarization domain. Recommender system uses opinions of other user to help individual user to identify information relevant to their needs or likely to interest [11]. We 
Vol. 5, Issue 12, December 2016

sometimes depend on the review in the paper to choose which place to visit, which to watch even we decide where to eat dinner at night. This provide shortcut to choose good service. Collaborative filtering is most famous algorithm to recommend product on the basis of user and item similarity. User does not know on which basis product is recommended [11]. Social network collect the large amount of data received from all over the world. This data contains number of rating and opinions about the product, mood and location details on the internet. This has important feature related to the product [15]. User's choice is always closely related to his/her personal interest. There are three important factors which is personal interest; interpersonal interest similarity and interpersonal influence are combined in to recommendation model based on matrix factorization technique [4]. [4] Mainly focus on the geo networking factors in probabilistic factorization model. Biases represent the habit of users rating such as user A ratings are mostly 5 and 4 and other user ratings are mostly 2 [4] . 'Inferred trust circle' based on the domain-obvious of circles of friends on social networks to recommend user favorite items [4]. To this user social user rating behavior is important for recommendation. First utilize user rating confidence by analyzing social user behaviors. Find users habits and taste. Extract the user rating confidence which is trustworthy ness of user rating by evaluation [5]. Trust values are computed by computing similarity measures between the users. This is evaluated by through process of trust calculation [11].

\section{B. Literature Review}

There has been lots of research done in the area of recommendation. Researchers started working on the problem of rating in the recommendation [1]. Gediminas Adomavicius and Alexander Tuzhilin presents overview of recommendation system, in which they are classified in to three parts such as content-based, collaborative, and hybrid recommendation. And give the limitation of existing recommendation system and ways to improve recommendation capabilities over user-item and contextual information [1].

Qi Liu, Enhong Chen, Hui Xiong Chris H. Q. Ding and Jian Chen examine the behavior of recommendation system and hidden request. Propose IExpand $=$ user interest expansion via personalized ranking approach. Main focused on item oriented model-based recommender system. There are three layer proposed under above approach user interest item for accurate ranking results. Deals with problem in existing recommendation approaches [2].

Xueming Qian He Feng, Guoshuai Zhao, Tao Mei determines the cold start problem and data sparsity problem of dataset. Based on the matrix factorization social factor are combined on single model, personal preferences, interpersonal similarity and interpersonal influence of rating. Proposed approach meets the individual user's choice. This system applied on Yelp Dataset [3][4].
In [5] state that user gives valuable feedback about product on the social sites and propose model of user service evaluation. In this system finds user rating confidence. Find trustworthiness of user ratings by calculating user rating confidence. Mining is applied on the spatio-temporal feature for finding user rating confidence.

Yehuda Koren, Robert Bell and Chris Volinsky examine the strategies of recommendations Content based filtering and item based filtering. Content based filtering makes user profiling, product to find its behavior. Means user profiling based on the content. It is not easy sometimes to collect data related to this. Collaborative filtering is another one which finds similarities between user and items and finds relation for recommendation item to user [6]. E.g. If user A given ratings to some movies and user $\mathrm{B}$ given ratings to some movies and both give ratings to common movies then A's movie recommended to B on the similarity basis. Michael Jahrer, Andreas Töscher, Robert Legenstein analyzes the state-of-the-art of collaborative filtering. Show the effectiveness of combined Collaborative filtering algorithms such as, SVD, Neighborhood approaches Restricted Boltzmann Machine, Asymmetric Factor Model and Global Effect. This ensembles blending give accurate prediction on $\mathrm{CF}$ [7].

Mohsen Jamali and Martin Ester in existing recommendation social relation considered to make recommendation. But they propose recommendation on model based, and matrix factorization. They focused on the trust propagation while recommendation. Trust based recommendation widely accepted in memory based approaches. Matrix factorization has been used for form model for recommendation. Use breadth first and shortest path algorithm for trust value calculation [8].

Yashar Moshfeghi, Benjamin Piwowarski and Joemon M. Jose address the problem of data sparsity in Collaborative Filtering. Propose framework for automatic to tackle problem of cold start problem by considering item related emotions and semantic data. Make final prediction using latent dirichlet and gradient boosted trees by extracting emotions [9].

In [10] presented Bayesian latent variable model. Latent topics and latent users interest model for rating prediction. Add Extension to this model by enhancing user dependent and item dependent biases. SVD is commonly used in this system.

\section{III.CONCLUSION}

In this paper, there is lots of work proposed on the personalized recommendation. In this lack of quality is service recommendation. It is important to provide quality of service on recommendation. Reviews also contain important information which will contain important feature to which is valuable to recommendation. In this system, we propose framework to provide quality of 
service recommendation by calculating trustworthiness of rating. First propose entropy model to calculate user rating trust and features extracted from the spatial and sentimental contextual information. And then combine all this to calculate overall trustworthiness.

\section{REFERENCES}

[1] G. Adomavicius and A. Tuzhilin, "Toward the next generation of recommender systems: a survey of the state-of-the-art and possible extensions," IEEE Trans. Knowledge and Data Engineering, vol. 17, no. 6, pp. 734-749, 2005.

[2] Q. Liu, E. Chen, H. Xiong, C. Ding, and J. Chen, "Enhancing collaborative filtering by user interest expansion via personalized ranking," IEEE Transactions on Systems, Man, and CyberneticsPart B (TSMCB), vol. 42, no. 1, pp. 218-233, 201.

[3] X. Qian, H. Feng, G. Zhao, and T. Mei, "Personalized Recommendation Combining User Interest and Social Circle," IEEE Trans. Knowledge and Data Engineering, vol. 26, no. 7, pp. 1487-1502, 2014

[4] H. Feng and X. Qian, "Recommendation via user's personality and social contextual," in ACM CIKM'13, pp. 1521-1524, 2013.

[5] G. Zhao and X. Qian, "Service objective evaluation via Exploring Social Users' Rating Behaviors," in Proceedings of the first IEEE International Conference on Multimedia Big Data, pp. 228-235, 2015.

[6] Y. Koren, R. Bell, and C. Volinsky, "Matrix factorization techniques for recommender systems," Computer, pp. 30-37, Aug. 2009.

[7] M. Jahrer, A. Toscher, and R. Legenstein, "Combining predictions for accurate recommender systems," in KDD'10, pp. 693- 702, 2010

[8] M. Jamali and M. Ester, "A matrix factorization technique with trust propagation for recommendation in social networks," in ACM RecSys'10, pp. 135-142, 2010

[9] Y. Moshfeghi, B. Piwowarski, and J. Jose, "Handling Data Sparsity in Collaborative Filtering using Emotion and Semantic Based Features," in SIGIR'11, pp. 625-634, 2011

[10] M. Harvey, M. Carman, I. Ruthven, and F. Crestani, "Bayesian latent variable models for collaborative item rating prediction," in CIKM'11, pp. 699-708, 2011

[11] P. Bedi, H. Kaur, and S. Marwaha, "Trust based recommender system for semantic web," in IJCAI'07, pp. 2677-2682, 2007.

[12] M. Jiang, P. Cui, R. Liu, Q. Yang, F. Wang, W. Zhu, and S. Yang, "Social contextual recommendation," in CIKM'12, pp. 45-54, 2012.

[13] X. Yang, T. Zhang, and C. Xu, "Cross-Domain Feature Learning in Multimedia," IEEE Trans. Multimedia, vol. 17, no. 1, pp.64-78, 2015

[14] Y. Zhang, B. Cao, and D. Y. Yeung, "Multi-domain collaborative filtering," in Proc.UAI, pp. 725-732, 2010

[15] G. Zhao, X. Qian, and X. Xie, "User-Service Rating Prediction by Exploring Social Users' Rating Behaviors," IEEE Trans. Multimedia, vol. 18, no. 3, pp. 496-506, 2016 